\title{
Current Non-Melanoma Skin Cancer Treatment Options
}

\author{
Neil Sandhu* \\ Daystar Skin and Cancer Center, USA
}

Submission: August 04, 2017; Published: August 30, 2017

*Corresponding author: Neil Sandhu, Medical Director for Daystar Skin and Cancer Center, USA, Email: sandhu.neil@gmail.com

\section{Introduction}

Non-melanoma skin cancer (NMSC) is the most commonly occurring cancer in the United States and in the world. 75-80\% of NMSCs are basal cell carcinomas (BCC). The remaining 25\% are squamous cell carcinomas (SCC). One in five Americans will develop skin cancer in their lifetime, of which more than $95 \%$ will be NMSCs. Over 2.1 million patients were treated for NMSCs in 2006. Despite its prevalence, NMSC accounts for less than $0.1 \%$ of cancer deaths.

NMSC is more common in the southern latitudes of the northern hemisphere. Cumulative exposure to ultraviolet (UV) radiation and the sensitivity of an individual's skin to UV radiation increase a patient's likelihood of developing NMSC. Patients with a light complexion who have accumulated significant exposure to sunlight throughout their lives are at a higher risk. SCCs tend to develop in patients who have experienced chronic, long-term ultraviolet (UV) radiation exposure. Conversely, individuals exposed to intermittent, intense UV radiation are more likely to develop BCCs. Additional factors that increase an individual's risk include prior psoralen and ultraviolet A(PUVA) treatment for psoriasis, exposure to ionizing radiation, and use of tanning lamps. Chemical exposures to pesticides, tar, polycyclic aromatic hydrocarbons, and arsenic increase the patient's risk for SCC. Serologic evidence from a population based case-control study has shown a possible association between NMSC and infection with human papilloma virus (HPV), particularly types 16, 18, and genus beta-species 1 [1]. Immunosuppressed patients, namely those receiving immunosuppressive drugs post organ transplantation, or patients with human immunodeficiency virus (HIV), chronic lymphocytic leukemia (CLL), or lymphoma are at an elevated risk of developing NMSC, specifically SCC.

Mutations of the tumor protein p53 are the most frequently altered genes implicated in the development of human cancers. These are usually missense mutations in DNA-binding domains preventing p53 from binding promoter regions on DNA. When an individual experiences chronic UV radiation, mutated epidermal p53 clones that are resistant to UV-induced apoptosis are formed, thereby creating a possible precursor for skin cancers including BCC and SCC. Protein patched homolog 1 (PTCH1) tumor suppressor gene mutations are the most common mutations seen in the development of BCC. PTCH1 genes are involved in cell cycle replication. Constitutive activation of signaling by PATCH inactivation or smoothened activation mutations are required for BCCs to develop.

BCCs arise from intermittent, recreational sun exposure and tend to be locally destructive, slow growing, and rarely metastasize. When metastasis does occur, BCCs spread via perineural invasion to lymph nodes, and can then progress to the lungs and bones. BCCs are most commonly seen in areas of the skin with pilosebaceous units, such as the nose. High-risk areas for tumor recurrence after initial treatment include the central face (e.g.., periorbital region, eyelids, nasolabial folds, or nosecheek angle), postauricular region, pinna, ear canal, forehead, and scalp. Clinical manifestations include elevation from surrounding skin, pearly quality, and presence of telangiectatic vessels.

Specific qualities of BCC lesions vary by type. Nodular type lesions are the most common; they are usually found on the face and may present with ulceration. Superficial BCCs usually appear on the trunk and extremities as an erythematous macule or thin plaque; atrophy or hypopigmentation may also be present. Morpheaform, or infiltrative, BCCs are flat, slightly atrophic lesions or plaques with poorly demarcated borders. They usually appear scar-like; telangiectasias may or may not be present. Cystic type BCCs are clear, blue-gray in color, and will exude clear fluid if ruptured. Fibroepitheliomas of Pinkus are a rare form of BCC that present on the lower back as smooth, pink plaques or nodules that may be pedunculated. Histology shows anastomosing strands of basal cells extending down from the epidermis. Micronodular is a histological term that can be used to describe smaller tumor aggregates that infiltrate the dermis. This finding may suggest a more aggressive BCC with higher likelihood of recurrence. Basosquamous carcinomas are the most aggressive and destructive subtype of BCCs.

SCCs are three times less common than BCCs in non-immunocompromised patients, but they tend to be more aggressive and have a varied range of growth, invasive and metastatic potential. 
Cumulative dose of UV radiation throughout the patients' life is a significant risk factor. Other predisposing factors include: Coal tar, arsenic, ionizing radiation; immunosuppression; chronic ulcers and scars (such as Marjolin's Ulcer), sites of prior burns, genetic syndromes (such as Xeroderma pigmentosum, or XP), and HPV types six, 11 (genital), and 16 (periungual). Cases of SCC arising from p53 missense mutations generally are diagnosed early in life. Ras oncogene activation mutations are seen in 10$15 \%$ of patients who present with SCC.

SCCs are most common on the face, hairless scalp, dorsal hands, and helices. Lesions that arise in areas of non-sunexposed skin or that originate de novo on areas of sun-exposed skin are prognostically worse as they have a greater tendency to metastasize than those occurring in sun-exposed skin or from preexisting actinic keratoses (AK). AK are most common in the head, neck, and upper extremities of the elderly due to chronic sun exposure. P53 clones lead to dysplasia which results in hyperkeratotic, pigmented, or lichenoid lesions; it can also manifest as actinic cheilitis or cutaneous horns. While AK are a precursor of SCC, only approximately $10 \%$ develop into SCC insitu over 10 years. Histologically, AK displays focal parakeratosis and partial thickness atypia.

SCC in-situ, or Bowen's disease, is a full-thickness dysplasia of the epidermis that presents as an erythematous scaly patch or plaque. Approximately 3-4\% of these lesions become invasive. Upon microscopic examination, diffuse confluent parakeratosis with full-thickness atypia over a broad zone will be present, along with frequent mitoses and apoptosis.

Invasive SCC can be classified as well, moderately, or poorly differentiated. Poorly- differentiated invasive SCC is progressive with overlapping features ending in highly infiltrative spindle cell tumors without overt keratinization. Cytokeratin stain is positive. S100 stain and CD68 marker are both negative. Moderatelydifferentiated invasive SCC may display acantholysis. Welldifferentiated invasive SCC will show the formation of keratin pearls and the presence of desmosomes between tumor cells.

Metastatic workup is generally not necessary after diagnosis of BCC, but if the lesion is suspicious for deep structural involvement, further testing should be obtained. MRI with contrast is preferred for perineural invasion and CT with contrast should be used to diagnose bone involvement. However, regional lymph nodes should be routinely examined in all cases of SCC, especially for high-risk tumors appearing on the lip, ears, medial canthi, perianal and perigenital regions, or high-risk areas of the hand. If palpable nodes are appreciated, then fine needle aspiration or core biopsy should be considered.

Despite their prevalence, NMSCs currently only account for $0.1 \%$ of cancer deaths. Surgical techniques, radiation, curettage and electrodessication (C \& E), and superficial therapies help decrease skin cancer mortalities. Surgical excision of wellcircumscribed lesions $<2$ centimeters $(\mathrm{cm})$ in diameter excised with 4-5 millimeters ( $\mathrm{mm}$ ) clinical margins results in complete removal in more than $95 \%$ of cases. Post-operative margin evaluation yields five-year disease- free rates of over $98 \%$ for both BCC and SCC. This includes re-excision of low-risk primary BCC located on the trunk and extremities, excluding pretibia, hands, feet, nail units, and ankles. High risk lesions require wider surgical margins $(6-10 \mathrm{~mm})$ and increased recurrence rates can be expected.

Mohs micrographic surgery (MMS), or excision with intra operative frozen section assessment, allows for intra operative analysis of $100 \%$ of the excision margin and is the preferred surgical technique for high-risk BCC/SCC. Five-year recurrence rates after MMS are 1.0\% for primary BCC, 3\% for SCC, and 5.6\% for recurrent BCC, lower than all other treatment modalities. Additionally, MMS provides the best cosmetic outcome as the least amount of tissue is excised to achieve clear margins. It is indicated in previously radiated skin, traumatic scars, in areas of osteomyelitis, areas of chronic inflammation or ulceration or in patients with genetic, syndromes regardless of location, depth or subtype. A prospective randomized trial comparing MMS to standard excision was completed in the Netherlands; the treatment of high-risk facial BCC with MMS resulted in fewer recurrences at 10-year follow-up when compared with standard excision, although the difference was only statistically significant for recurrent tumors. A large proportion of recurrences occurred more than five years after treatment: $56 \%$ for primary and $14 \%$ for recurrent BCC, highlighting the need for long-term follow-up.

MMS is indicated for the treatment of BCC, including recurrent, primary aggressive, primary nodular and primary superficial on the face, genitalia, hands, feet, nail units, ankles, nipples/areola, cheeks, forehead, scalp, neck, jawline and pretibial surfaces regardless of size or patient type. However, it is not indicated for primary superficial BCC lesions $0.5 \mathrm{~cm}$ or smaller on the cheeks, forehead, scalp, neck, jawline and pretibial surfaces for healthy patients. For other areas on trunk and extremities, appropriate use of Mohs surgery depends on type and size of the BCC lesion.

MMS is indicated for all high-risk SCC cases which are listed as sclerosing, basosquamous, poorly or undifferentiated, perineural or perivascular, spindle cell, pagetoid, infiltrating, keratoacanthoma type, including: central facial, single cell, clear cell, lymphoepithelial, or sarcomatoid. MMS is also appropriate for Breslow depth of $2 \mathrm{~mm}$ or greater and Clark level IV or greater. It is not suggested for treatment of AK with focused SCC in situ, Bowenoid AK or SCC in situ of AK subtype. MMS may not be beneficial for treatment of primary SCC in areas of the trunk and extremities when it is not an aggressive subtype. Lesions $<2 \mathrm{~mm}$ in depth and $<2 \mathrm{~cm}$ in size in healthy patients or $<1 \mathrm{~cm}$ in size in immunocompromised patients may not be appropriate for MMS [2]. The Appropriate Use Criteria developed by American Academy of Dermatology is available in application form for ease of access.

Radiation therapy represents another treatment option for patients with NMSC. 5-year recurrence rates of $8.7 \%$ and $10 \%$ are shown after radiation therapy on primary and recurrent BCC 
and SCC, respectively. 5-year local control, cure, or complete response rates range from $93 \%$ to $96 \%$. However, studies have shown higher recurrence rates $(7.5 \%$ vs. $0.7 \%$; $\mathrm{P}=.003)$, poorer cosmetic outcomes, and more postoperative complications when compared with excisional surgery. Efficacy was better for BCCs that were less advanced, primary (vs. recurrent), or of nodular histologic subtype. Perineural invasion is associated with poorer outcomes, therefore adjuvant radiotherapy is recommended when the lesion involves more than a few small sensory nerve branches or has large nerve involvement $(>0.1 \mathrm{~mm})$, even if tumor is resected and margins are clear. Adjuvant surgical excision is also appropriate with recurrent or high-risk SCC that is $>2 \mathrm{~cm}$, has ill-defined margins, has depth $>4 \mathrm{~mm}$, is poorly differentiated or shows rapid growth [3]. Radiation therapy may be considered if positive tissue margins are present after MMS as local control approaches $100 \%$ with the use of postoperative radiotherapy. Radiation therapy is contraindicated in patients with genetic conditions predisposing to skin cancer (e.g., basal cell nevus syndrome, xeroderma pigmentosum) and those with tissue diseases (e.g., lupus, scleroderma). Often, radiation therapy for NMSC is reserved for patients older than 60 years because of concerns about long-term sequelae

Superficial radiation therapy (SRT) uses low energy kilo voltage photons $(50-150 \mathrm{kVp})$ to deliver electromagnetic energy to rapidly dividing cells, thereby stopping mitosis. Dosing is based on cGY (Gray) units and is influenced by the tumor size and age of the patient. Treatment is typically given over 1317 sessions, or fractions. The recurrence rate of SRT has been shown to be between $1.9-5 \%$ for NMSC. This type of treatment is recommended for patients who are older ( $>70$ years), have significant medical comorbidities, use anticoagulants, or simply prefer not to have surgery. Ala nasi, nasal tip, nasal bridge, eyelid, medial canthi and helices are ideal sites for SRT use. Acute reactions include erythema and mild discomfort. Patients can expect a healing time of approximately four weeks after completion of treatment. Late reactions include hyper or hypopigmentation, telangiectasias, atrophy, and ulcers.

Curettage and electrodessication (C\&E) consists of alternatively scraping away tumor tissue with a curette down to a firm layer of normal dermis and denaturing the area by electrodessication. Up to three cycles may be performed per session. This treatment method does not allow for histologic margin assessment and therefore is best for low-risk tumors. Results are highly-operator dependent. Overall five-year cure rates range from $91 \%$ to $97 \%$ in patients with BCC selected for C\&E. Higher recurrence rates (19\%-27\%) have been reported with high-risk locations and more aggressive histologic subtypes. Research cases show that tissues taken through C\&E have 25\% persistent BCC upon histology examination. C\&E should not be used to treat areas with terminal hair growth, as tumors extending down follicular structures may not be adequately removed. If the subcutaneous layer is reached during C\&E, then surgical excision should be performed. This is due to the diminished ability of the curette to distinguish and to selectively remove tumor cells completely since subcutaneous adipose tissue is softer than tumor tissue. Biopsy results of the tissue taken at time of curettage should routinely be reviewed to make sure that there are no high-risk features.

Superficial therapies typically result in low cure rates and therefore should be reserved for those patients in whom surgery or radiation is contraindicated or impractical. Photodynamic therapy can be used for superficial BCC, nodular BCC, and SCC in situ. A photosensitizing agent is applied on the skin, allowed to incubate under occlusion for four to six hours, then followed by irradiation with a light source (e.g. 630nm). Photosensitizing agents include methyl aminolevulinate (MAL) for deep lesions and five-aminolevulinic acid (ALA) for superficial lesions. Treatment can be repeated four weeks later as needed. Photodynamic therapy is more likely to produce good cosmetic outcomes when compared to surgery, but cure rates are much lower (70-90\%), especially in lesions with ulceration or of deeper depth. Cryotherapy uses liquid nitrogen at $-195.6{ }^{\circ} \mathrm{C}$ to cause intra and extracellular ice formation, which destroys tissues via cell dehydration [4]. Recurrence rates range from $5 \%$ to $39 \%$. The variability of recurrence rates may be due to patient selection, variable follow-up durations, and differences in operator skill and technique. Cryosurgery generally has poor cosmetic outcomes does not provide histological evidence of tumor removal.

Imiquimod modifies the immune system to stimulate toll-like receptor seven. It has been approved for treatment of superficial BCC and AK. Treatment regimen most commonly consists of once daily application, five times weekly for six weeks. Use of imiquimod for superficial and nodular BCC showed an $84 \%$ clinical success rate, defined as absence of initial treatment failure or signs of recurrence at 3 years from start of treatment. This treatment response continued to include an $85 \%$ fiveyear disease-free rate in superficial BCC. While clinical success rates were significantly higher in patients treated with surgical excision using a $4 \mathrm{~mm}$ margin $(98 \%, \mathrm{P}<.001)$, cosmetic outcomes were significantly better with imiquimod (excellent/good at three-year follow-up: $61 \%$ vs. $36 \% \mathrm{P}<.0001$ ) [5].

Topical fluorouracil (5-FU) is also used to treat superficial BCC and AK. 5-FU works as a thymidylate synthase (TS) inhibitor that blocks synthesis of the pyrimidine thymidine to decrease DNA damage. It has been shown in a randomized trial to have similar efficacy, safety, and cosmetic outcomes as imiquimod. Most commonly, treatment recommendations include application one or two times daily for three to six weeks. Nonsurgical treatment selection depends on many factors, and each option should be carefully weight against the others. For patients with superficial BCC, photodynamic therapy (PDT) has similar efficacy as cryotherapy but has much better cosmetic outcomes. In those same patients, PDT, imiquimod, and fluorouracil all have similar efficacy and cosmetic outcomes. Treatment success rates are significantly higher with imiquimod versus PDT for tumors that are large or truncal. For elderly patients with lesions on the 
lower extremities, PDT provided significantly better outcomes than imiquimod. Combination therapy can also enhance cure rates and maximize cosmesis. 5-FU plus imiquimod is an effective combination to pretreat BCC before performing MMS or to treat SCC in situ in transplant patients. BCC curettage can be followed by imiquimod five times weekly for six weeks to maximize desirable outcomes.

Intralesional and systemic chemotherapy are also available as treatment options. When compared to excision and MMS, BCCs injected with interferon alpha $2 \mathrm{a}$, interferon alpha $2 \mathrm{~b}$, interferon gamma, interferon beta, bleomycin and 5-FU provided inferior complete response rates [6]. Delivery of chemotherapy can be enhanced through electrochemotherapy, which uses electric pulsations to temporarily increase the permeability of cells to the drug. A case series of 25 patients using electrochemotherapy with bleomycin to treat cutaneous and subcutaneous NMSC of the head and neck were found to have a $72 \%$ complete response rate with no recurrence during 18 months of follow-up. A complete response was observed in $100 \%$ of BCCs and $65 \%$ of SCCs [7].

For patients with BCC nevoid syndrome, locally advanced BCC that has recurred following surgery or radiation, or those who are not candidates for surgery or radiotherapy, systemic therapy can be utilized. The hedgehog pathway inhibitors Vismodegib and Sonidegib can significantly reduce the incidence of new BCC lesions, the size of existing lesions, and the number of surgeries needed to remove BCC lesions. Resistance is common with the use of these systemic therapies. Metastases are seen in approximately five percent of SCCs arising chronically sunexposed skin. The rate of metastasis increases to $30 \%$ with mucocutaneous SCCs. Metastatic risk increases with tumor size, location, depth, differentiation, perineural involvement, immune-suppression, recurrence, or location in a chronic scar. Ten-year survival with distant metastases is $10 \%$. Factors that increase the risk of nodal metastasis include lymphovascular invasion, poor differentiation, and perineural invasion. Treatment for nodal metastasis includes lymph node dissection plus adjuvant radiation therapy with or without concurrent chemotherapy. Parotid involvement, as direct extension from an overlying cutaneous SCC, is a poor prognostic factor. If invasion is present into the parotid fascia, a superficial parotidectomy needs to be performed. BCC with nodal or distant metastases should be treated with surgery, with or without radiation therapy, and managed by a multidisciplinary tumor board. With metastatic SCC, cisplatin, either as a single agent or combined with 5-FU, has occasionally produced useful responses, but data supporting efficacy is limited.

Emerging treatments show promise for the management of NMSC. Cryoimmunotherapy for BCCs and SCC in situ combines cryotherapy with imiquimod cream. The cryotherapy- mediated damage to the stratum corneum facilitates penetration of imiquimod, leading to a recurrence rate of only $2 \%$ [8]. Ingenolmebutate is a purified compound from the sap of Euphorbia peplus plant. Tumor control is achieved through primary necrosis followed by an inflammatory response targeting residual tumor cells. A two to three-day course yields 100\% histologic clearance $(n=9)$ [9]. The deoxyribozyme, Dz13, can be used to decrease expression of c-Jun, to alter transcription factor formation. When injected into nodular basal cell carcinomas in nine patients, no serious drug-related side effects were apparent and histological tumor depth decreased in more than half of patients [10-12].

Due to its immense prevalence, effective treatments for NMSC are essential. Surgery and radiotherapy appear to be the most effective treatments. Surgical excision, particularly MMS, provides the most effective treatment with regards to recurrence rates and cosmetic outcomes. Non-surgical treatments may be considered as appropriate, including radiation therapy, C\&E, PDT, cryotherapy, topical medications, and intralesional chemotherapy. These treatments have benefits and associated risks but are generally associated with higher recurrence rates, inferior cosmetic outcomes, and increased complications when compared to surgical excision. Combination therapy should be considered for higher- risk lesions to achieve additional control. Management of metastasis includes surgery, lymph node dissection, radiation, and/or chemotherapy. While more research is needed and patients inevitably require long-term surveillance for recurrence or new lesions, the above treatment methods have given practitioners the ability to effectively treat and control NMSCs.

\section{References}

1. Patel AS, Karagas MR, Perry AE, Nelson HH (2008) Exposure profiles and human papilloma virus infection in skin cancer: an analysis of 25 genus betatypes in a population based study. J Invest Dermatol 128(12): 2888-2893.

2. Gargiulo M, Papa A, Capasso P, Molo M, Cubicciotti E, et al. (2012) Electrochemotherapy for non-melanoma head and neck cancer: Clinical outcomes in 25 patients. Ann Surg 255(6): 1158-1164.

3. Hulyalkar R, Rakkhit T, Garcia-Zuazaga J (2011) The role of radiation therapy in the management of skin cancers. Dermatol Clin 29(2): 287296.

4. Kirby JS, Miller CJ (2010) Intralesional chemotherapy for nonmelanoma skin cancer: a practical review. J Am Acad Dermatol 63(4): 689-702.

5. Bath HF, Ozolins M, Armstrong SJ, Colver GB, Perkins W, et al. (2014) Surgical excision versus imiquimod $5 \%$ cream for nodular and superficial basal-cell carcinoma (SINS): A multicentre, non-inferiority, randomised controlled trial. Lancet Oncol 15(1): 96-105.

6. Marks JG, Miller JJ (2013) Lookingbill and Marks' Principles of Dermatology. ( $5^{\text {th }}$ edn), Saunders Elsevier, USA.

7. Connolly SM, Baker DR, Coldiron BM, Fazio MJ, Storrs PA, et al. (2012) AAD/ACMS/ASDSA/ASMS 2012 appropriate use criteria for Mohs micrographic surgery: a report of the American Academy of Dermatology, American College of Mohs Surgery, American Society for Dermatologic Surgery Association, and the American Society for Mohs Surgery. J Am Acad Dermatol 67(4): 531-550. 
8. MacFarlane DF, El Tal AK (2011) Cryoimmunotherapy: superficial basal cell cancer and squamous cell carcinoma in situ treated with liquid nitrogen followed by imiquimod. Arch Dermatol 147(11): 13261327.

9. Bettencourt MS (2016) Treatment of superficial basal cell carcinoma with ingenolmebutate gel, $0.05 \%$. Clin Cosmet Investig Dermatol 16(9): 205-209.

10. Cho EA, Moloney FJ, Cai H (2013) Safety and tolerability of an intratumorally injected DNAzyme, Dz13, in patients with nodular basal-cell carcinoma: A phase 1 first-in-human trial (DISCOVER). Lancet 381(9880): 1835-1843.

11. Rogers HW, Weinstock MA, Harris AR, Hinckley MR, Feldman SR, et al. (2006) Incidence estimate of nonmelanoma skin cancers in the United States. Arch Dermatol 146(3): 283-287.

12. https://www.nccn.org/professionals/physician_gls/pdf/nmsc.pdf

\section{Your next submission with Juniper Publishers will reach you the below assets}

- Quality Editorial service

- Swift Peer Review

- Reprints availability

- E-prints Service

- Manuscript Podcast for convenient understanding

- Global attainment for your research

- Manuscript accessibility in different formats

( Pdf, E-pub, Full Text, Audio)

- Unceasing customer service

Track the below URL for one-step submission https://juniperpublishers.com/online-submission.php 